\title{
THE COSMIC MICROWAVE BACKGROUND ANISOTROPIES: OPEN PROBLEMS
}

\author{
Enrique Martínez-González \\ Instituto de Física de Cantabria \\ Consejo Superior de Investigaciones Científicas-Universidad de Cantabria \\ martinez@ifca.unican.es \\ Patricio Vielva \\ Instituto de Física de Cantabria \\ Consejo Superior de Investigaciones Científicas-Universidad de Cantabria \\ vielva@ifca.unican.es
}

Keywords: Cosmic Microwave Background, data analysis, cosmological parameters

\begin{abstract}
We present a review of some interesting theoretical and observational aspects of the Cosmic Microwave Background anisotropies. The standard inflationary model presents a simple scenario within which the homogeneity, isotropy and flatness of the universe appear as natural outcomes and, in addition, fluctuations in the energy density are originated during the inflationary phase. These seminal density fluctuations give rise to fluctuations in the temperature of the Cosmic Microwave Background (CMB) at the decoupling surface. Afterward, the $\mathrm{CMB}$ photons propagate almost freely, with slight gravitational interactions with the evolving gravitational field present in the large scale structure (LSS) of the matter distribution and a low scattering rate with free electrons after the universe becomes reionized by the first stars and QSOs. These secondary effects slightly change the shape of the intensity and polarization angular power spectra of the radiation, the so called $C_{\ell}$. The $C_{\ell}$ contain very valuable information on the parameters characterizing the background model of the universe and those parametrising the power spectra of both matter density perturbations and gravitational waves. The extraction of this richness of information from the $C_{\ell}$ is complicated by the superposition of the radiation coming from other Galactic and extragalactic emissions at microwave frequencies. In spite of this, in the last few years data from sensitive experiments have allowed a good determination of the shape of the $C_{\ell}$, providing for the first time a model of the universe very close to spatially flat. In particular the WMAP first year data, together with other CMB data at higher resolution and other cosmological data sets, have made possible to determine the cosmological parameters with a precision of a few percent. The most striking aspect of the derived model of the universe is the unknown nature of most of its energy contents. This and other open problems in cosmology
\end{abstract}


represent exciting challenges for the CMB community. The future ESA Planck mission will undoubtely shed some light on these remaining questions.

\section{Introduction}

In recent years there has been an explosion of cosmological data allowing a strong progress in the characterization of the cosmological model of the universe. In particular, recent data of the temperature of the Cosmic Microwave Background (CMB) have played a crucial role in the determination of the cosmological parameters. Many experiments aimed to map the temperature of the CMB have been and are being carried out, and many others are now being planned to extend present capabilities in resolution and sensitivity. From the cosmological data already collected we know that the spatial geometry of the universe is close to Euclidean, with most of the energy density in the form of the so called "dark energy (DE)" (with an equation of state close to a cosmological constant) and most of the matter density in the form of the so called "cold dark matter (CDM)" (weakly interactive matter with negligible velocity). About the primeval density perturbations we know that they were close to the adiabatic type (constant ratio of the matter number density to photon density for each matter component) and Gaussianly distributed with a nearly scale-invariant power spectrum (i.e. the gravitational potential fluctuations are the same at all scales). The generic predictions of inflation - a very brief episode of drastic expansion in the very early history of the universe close to the Planck time - are consistent with the characteristics of the model of the universe and of the primeval density fluctuations just mentioned, the so called "concordance model". Thus, inflation provides us with a plausible scenario within which we can understand the horizon and flatness problems (i.e. why causally disconnected regions appear statistically similar in the CMB sky and why the density of the universe is so close to the critical one) and also the origin of the density perturbations. Although a physical model of inflation is still lacking, however, it provides us with a phenomenological scenario within which we can conceptually understand some fundamental problems related to the origin of the "special" properties of the universe -i.e. its homogeneity and critical density - and its large scale structure (LSS) matter distribution which otherwise will have to rely on ad hoc initial conditions. In the past decade two of the experiments on-board of the NASA COBE satellite, FIRAS and DMR, established unambiguously the black-body electromagnetic spectrum of this radiation (Mather et al. 1994, 1999) and the level and approximate scaleinvariant shape of the spectrum of density fluctuations (Smoot et al. 1992). The former data rebated some previous results which indicated possible distortions from the black-body spectrum, establishing the thermal origin of the 
$\mathrm{CMB}$ with a high precision. The latter confirmed the gravitational instability theory for the formation of the LSS and determined an initial spectrum of fluctuations characterized by density fluctuations with approximately equal amplitude when entering the horizon. These fundamental results set the basis for the later developments in our understanding of the universe and opened the era of precision cosmology. At the end of the decade several experiments determined that the universe is close to spatially flat (BOOMERANG, De Bernardis et al. 2000; MAXIMA, Hanany et al. 2000). More recently the NASA WMAP satellite (Bennett et al. 2003) nicely confirmed this result and, together with other higher resolution CMB experiments as well as the galaxy survey 2dFGRS (Percival et al. 2001), determined the cosmological parameters with a few percent errors. The combination of different cosmological data sets not only helps to improve the precision of the cosmological parameters but, what is more important, shows the compatibility of the different data sets in the context of the concordance model. The most important features of this model is the distribution of the energy content of the universe with about $70 \%$ of dark energy, $25 \%$ of cold dark matter (CDM) and only $5 \%$ of baryons. The detection of the polarization fluctuations by DASI (Kovac et al. 2002) and the later determination of the temperature-polarization angular cross-power spectrum by WMAP (Kogut et al. 2003) has strongly confirmed the concordance model. Very recently, there have been several works finding evidences of positive cross-correlations between the CMB temperature map and the LSS distribution of galaxies (see e.g. Boughn and Crittenden 2004). These results represent an independent piece of evidence of the existence and dominance of dark energy in the recent history of the evolution of the universe (assuming that the universe is close to flat). Although the concordance $\Lambda \mathrm{CDM}$ model represents a good fit to the CMB data as well as to other cosmological data sets - namely LSS galaxy surveys, primordial Big-Bang nucleosynthesis, measurements of the Hubble constant, SN Ia magnitude-redshift diagram - there are however some problems related to the observations and also to their interpretation.

The aim of this paper is to review some of the most relevant theoretical and observational results on the CMB field and to point out some open problems associated to them. For more detailed discussions the reader is refereed to the many good reviews written in the literature, some of them more oriented to the theory (e.g. Hu and Dodelson 2002, Challinor 2004, 2005, Cabella and Kamionkowski 2004) and others to the experiments (e.g. Barreiro 2000, Bersanelli, Maino and Mennella 2002, Mennella et al. 2004).

The rest of this review is as follows. In Section 1 we describe the main properties of the CMB temperature anisotropies and the physical effects that originate them. In Section 2 the most relevant properties of the CMB polarization anisotropies are considered. The cosmological parameters are defined in Section 3 and their values determined from recent $\mathrm{CMB}$ data and in combination 


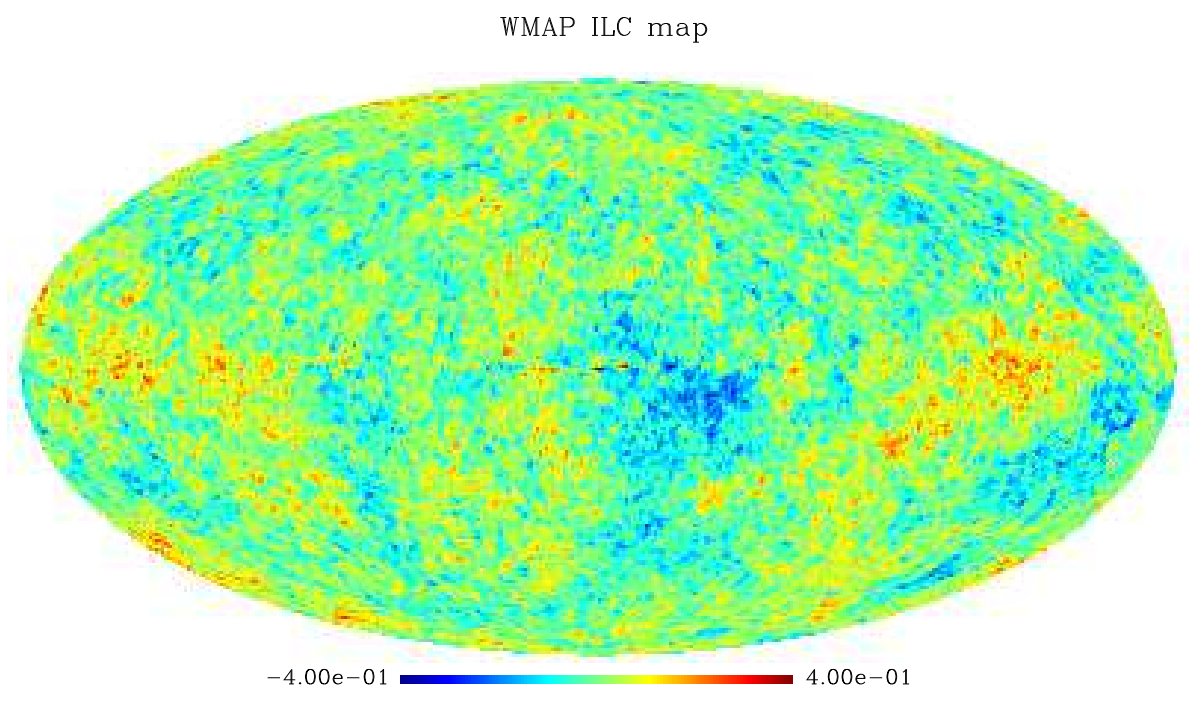

Figure 1. The CMB as seen by WMAP (data obtained from the LAMBDA wave page of NASA).

with other cosmological data sets are presented in Section 4. Finally, open problems in the CMB field are discussed in Section 5.

\section{CMB temperature anisotropies}

\section{Description}

The anisotropies of the CMB - the fluctuations in the intensity of this radiation as a function of the direction in the sky - are interpreted as a realization of a random field on the sphere. Behind this interpretation is the idea that we aim to understand and possibly explain the anisotropies in a statistical manner. Because of the blackbody spectrum of the CMB, the anisotropies in the intensity are generally given as temperature ones. Since the temperature fluctuations are a function of the spherical coordinates it is convenient to expand them in spherical harmonics,

$$
\frac{\Delta T}{T}(\vec{n})=\sum_{\ell, m} a_{\ell m} Y_{\ell m}(\vec{n})
$$

where $a_{\ell m}$ are the spherical harmonic coefficients. A very important quantity is the CMB anisotropy angular power spectrum, $C_{\ell}$, the second order moment 
of the $a_{\ell m}$ defined as

$$
<a_{\ell m} a_{\ell^{\prime} m^{\prime}}^{*}>=C_{\ell} \delta_{\ell \ell^{\prime}} \delta_{m m^{\prime}} .
$$

The null correlation of the harmonic coefficients for different $\ell$ or $m$ is due to the homogeneity and isotropy of the universe - i.e. the Cosmological Principle assumed as a fundamental pillar in cosmology and consistent with all of our observations up to date. Here it is important to notice, however, that the previous equation does not hold if the universe possesses a nontrivial topology. An important property of the $C_{\ell}$ is that if the anisotropies are Gaussian - as predicted by Inflation - then all the statistical information is contained in it (reported deviations from Gaussianity will be discussed in section 5). The correlation function of the temperature fluctuations, $C(\theta) \equiv<\Delta T / T\left(\vec{n}_{1}\right) \Delta T / T\left(\vec{n}_{2}\right)>$ with $\vec{n}_{1} \vec{n}_{2}=\cos (\theta)$, is related to the $C_{\ell}$ through the Legendre transform

$$
C(\theta)=\sum_{\ell} \frac{2 \ell+1}{4 \pi} C_{\ell} P_{\ell}(\cos \theta) .
$$

The isotropy of the field is now reflected in the independence of the correlation from the direction. Although the two quantities $C(\theta)$ and $C_{\ell}$ contain the same information, however the null correlation of the $C_{\ell^{\prime}}$ for different values of $\ell$ makes the latter quantity preferable for cosmological studies. The quantity which is usually displayed is $\ell(\ell+1) C_{\ell} / 2 \pi$, i.e. the power per logarithmic interval in $\ell$ for large $\ell$. Since the $\ell=1$ moment is dominated by our motion only moments with $\ell \geq 2$ are considered.

There is a fundamental limitation in the accuracy with which the angular power spectrum $C_{\ell}$ can be determined due to the fact that we can only observe one last-scattering surface - the error associated to it is called "cosmic variance". Since there are $2 \ell+1 a_{\ell m}$ coefficients for a given $\ell$ then for Gaussian temperature fluctuations the cosmic variance is easily calculated (from the dispersion of a chi-squared distribution with $2 \ell+1$ degrees of freedom)

$$
\Delta C_{\ell}=\frac{1}{\sqrt{\ell+0.5}} C_{\ell} .
$$

There are other sources of error which should be added to the cosmic variance. One is the fraction of the sky $f_{s k y}$ covered by an experiment, which increases the error by a factor $f_{s k y}^{-1 / 2}$ (Scott et al. 1994). Another one is the sensitivity of the experiment whose noise power spectrum adds to the $C_{\ell}$ of the cosmic signal in equation 4. Finally, there is a source of error coming from the process to separate the cosmic signal from the other foregrounds, namely Galactic emissions $^{1}$ (synchrotron, free-free and thermal dust), extragalactic sources, galaxy

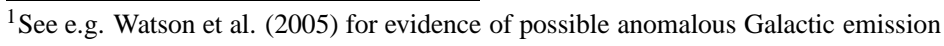


clusters and the lensing effect from the LSS. This is usually a complex task to perform which requires multifrequency observations and whose error is difficult to estimate a priori. Estimate of the errors assuming different situations and methodology can be found in, e.g., Hobson et al (1998), Tegmark et al. (2000), Bouchet and Gispert (1999), Vielva et al. (2001), Maino et al. (2002), Herranz et al. (2002), Martínez-González et al. (2003), Delabrouille et al. (2003).

In figure 1 the map of temperature anisotropies as measured by WMAP is shown (Bennett et al. 2003). Although a large effort has been made to eliminate all foreground contributions however some residuals from Galactic foregrounds can still be seen in the central horizontal band of this map. In order to avoid the introduction of this contamination in the data analysis a mask covering that and other regions of the map is usually applied.

\section{Physics}

The CMB anisotropies are usually divided in primary and secondary, depending if they are produced before or after the last-scattering surface. The primary anisotropies are the most interesting ones to study the cosmological parameters characterising the universe as well as its basic matter and energy constituents. The secondary ones are produced by scattering of the CMB photons from ionized matter - either generated after the reionization of the universe or present as hot gas in the central regions of galaxy clusters and whose interaction with the CMB photons is known as the Sunyaev-Zeldovich effect (SZ, Sunyaev and Zeldovich 1972)—, and by gravitational interactions acting on them during their travel from the last-scattering surface to the observer - causing both redshift and lensing effects. They are expected to be sub-dominant, at least in relation to the $C_{\ell}$, up to $\ell \approx 2500$.

The main physical effects producing the primary anisotropies and observed in the synchronous-comoving gauge (the observer's gauge) can be summarized in the following equation (Martínez-González, Sanz and Silk 1990, Sanz 1997):

$$
\frac{\Delta T}{T}(\vec{n}) \approx \frac{\phi_{e}(\vec{n})}{3}+\int_{e}^{o} \frac{\partial \phi}{\partial t} d t+\vec{n} \cdot\left(\vec{v}_{o}-\vec{v}_{e}\right)+\left(\frac{\Delta T}{T}(\vec{n})\right)_{e}
$$

The first two terms in the r.h.s. account for the gravitational redshift suffered by the CMB photons in their travel toward us along the direction $\vec{n}$. They are called the Sachs-Wolfe (SW) and Integrated Sachs-Wolfe (ISW) effects, respectively (Sachs and Wolfe 1967). The third term is a Doppler effect due to the motion of the emitters at the last-scattering surface. Finally the fourth term is the temperature fluctuation at that surface.

Since the gravitational field has a large scale of interaction the SW and ISW effects dominate the angular power spectrum at the largest angular scales $(\ell$ 


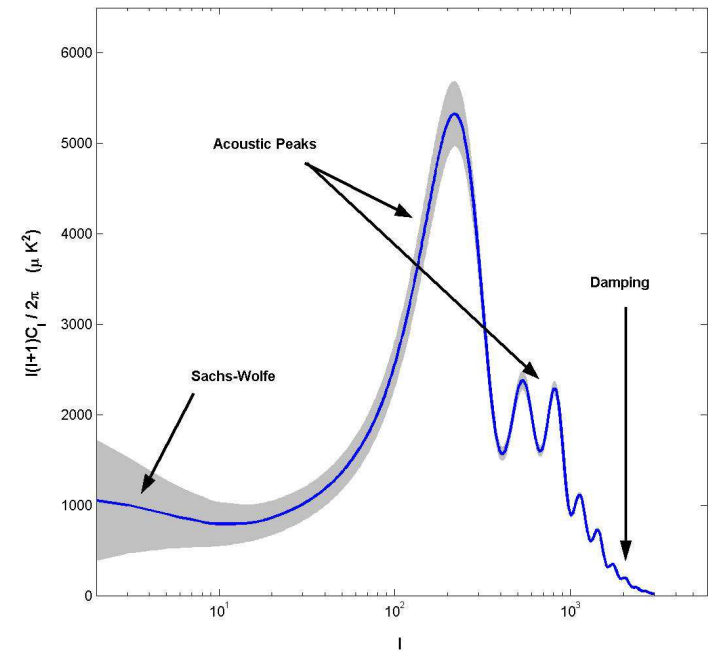

Figure 2. $C_{\ell}$ for the best fit model given in Bennett et al. (2003) (see also table 2). The range of $\ell$ where the different physical effects dominate is indicated. The gray band envolving the $C_{\ell}$ is the unavoidable error due to the cosmic variance (see text). The spectrum has been computed using the CMBFAST code (Seljak and Zaldarriaga 1996).

smaller than a few tens) producing an approximate plateau (see figure 2). Notice also that these scales are the most affected by the cosmic variance (see equation 4). The ISW effect has two contributions: an early one before recombination produced by the imperfect coupling of photons and baryons causing variations in the gravitational potential with time, and a late one after recombination due to changes in the gravitational potential with time. The late ISW effect is produced by the linear evolution of the large-scale matter distribution at later times if the universe is different form Einstein-de Sitter. As we will see at the end of Section 4 this is an independent test from the standard one (involving usually CMB, SN Ia or LSS data sets) about the existence of a dark energy component dominating the dynamics of the universe at recent cosmic times.

The Doppler effect plus the temperature fluctuations at the last-scattering surface dominate the shape of the angular power spectrum at $\ell$ larger than a few tens. At multipoles above a hundred, the angular power spectrum exhibits a sequence of oscillations called acoustic peaks. They are driven by the balance between the gravitational force pulling to compress over-dense regions and radiation pressure pushing in the opposite direction. The position and amplitude of these oscillations are very much determined by the total energy content of 

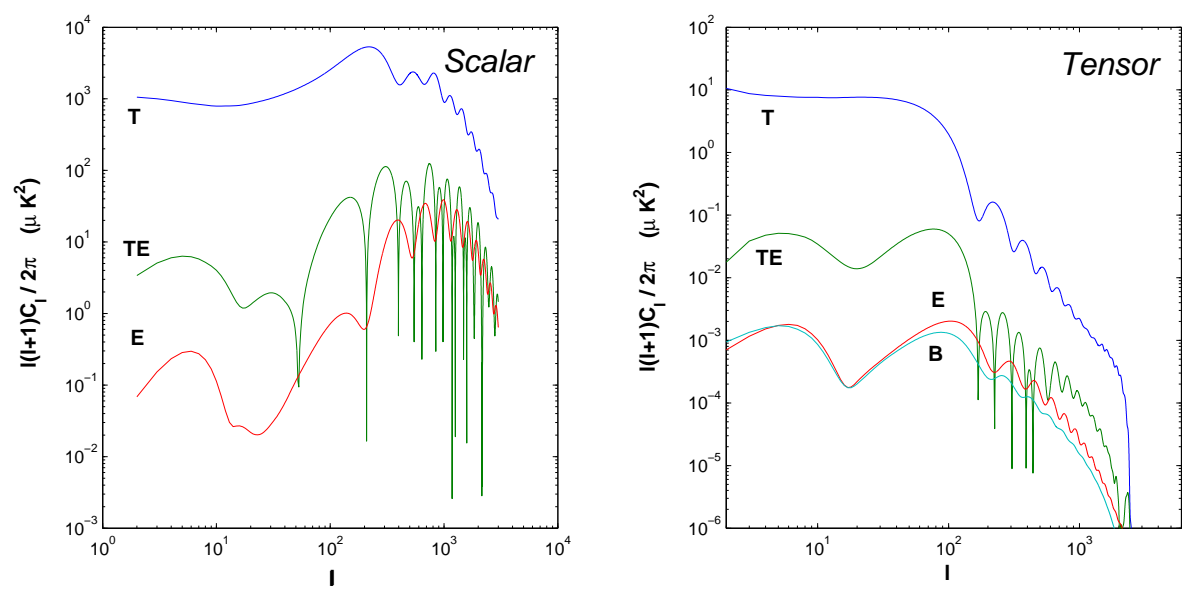

Figure 3. Temperature and polarization angular power spectra for scalar and tensor perturbations. The ratio tensor/scalar has been chosen to be $r=0.01$. Apart from this parameter, the vlues for the rest of the parameters have been fixed to the values of the best fit model given in Bennett et al. 2003 (see also table 2). All the spectra have been computed using the CMBFAST code.

the universe (or equivalently its geometry) and the nature and amount of the different components.

Finally, at large $\ell(\gtrsim 1000)$ the $C_{\ell}$ power starts to decrease due to the width of the last-scattering surface and the imperfections of the coupling of the photonbaryon fluid (Silk effect, Silk 1968).

In Figure 2 one can see the range of $\ell$ where the different effects dominate the angular power spectrum.

\section{Polarization}

Thomson scattering of the radiation generates linear polarization at the end of recombination when the growth of the mean free path of the photons allow anisotropies to grow (for a detailed description of the physics of polarization see, e.g., Challinor 2005). The expected level of polarization is only of $\approx 5 \%$. From the Stokes parameters $Q, U$ two rotationally invariant quantities can be constructed $E, B$ (often referred to as the E-mode and B-mode, see Zaldarriaga and Seljak 1997, Kamionkowski et al. 1997). Under parity transformations $E$ remains unchanged and $B$ changes sign, and therefore the cross-power $<a_{\ell m}^{B} a_{\ell m}^{T *}>=<a_{\ell m}^{E} a_{\ell m}^{B *}>=0$. It is for these parity properties that only three angular power spectra are required to characterise $\mathrm{CMB}$ polarization: $C_{\ell}^{E}, C_{\ell}^{B}, C_{\ell}^{T E}$.

An important property of the E,B decomposition is that whereas the E-mode polarization can be generated by both density perturbations and gravitational 
waves the B-mode can only be generated by the second ones. Therefore the detection of B-mode polarization is a unique proof of the existence of primordial gravitational waves, opening the door to new areas of physics. In particular, it can also be used to impose strong constraints on the energy scale of inflation and on the shape of the inflaton potential. A complication comes from the lensing effect due to the gravitational potential of the LSS which converts part of the E-mode polarization in B-mode. Fortunately, this effect dominates over the primary one only at $\ell \gtrsim 100$ leaving the rest of the spectrum unaltered. All the temperature and polarization angular power spectra for scalar and tensor perturbations are plotted in figure 3 .

As for the temperature angular power spectrum, the accuracy in the determination of the polarization angular power spectra has also a fundamental limit imposed by the cosmic variance. For Gaussian distributed anisotropies the errors in $C_{\ell}^{E}, C_{\ell}^{B}, C_{\ell}^{T E}$ are given by:

$$
\Delta C_{\ell}^{E, B}=\frac{1}{\sqrt{\ell+0.5}} C_{\ell}^{E, B}, \quad \Delta C_{\ell}^{T E}=\frac{1}{\sqrt{\ell+0.5}}\left(C_{\ell}^{T} C_{\ell}^{E}+C_{\ell}^{T E^{2}}\right)^{1 / 2} .
$$

There are a number of Galactic and extragalactic foregrounds which complicate the observation of the CMB polarization. Although their relevance depends very much on the frequency they are expected to be very harmful, specially for the B-mode due to its relatively small amplitude. For a recent estimate of the effect of foregrounds on the polarization observations see Tucci et al. (2005).

Only recently the DASI experiment has been able, for the first time, to detect anisotropies in polarization (Kovac et al. 2002). Afterward, the WMAP experiment measured the TE angular cross-power spectrum with more precision and covering a much wider range of scales (Kogut et al. 2003). More recently the $\mathrm{CBI}$ experiment has measured the $\mathrm{E}$ angular power spectrum with more resolution than DASI allowing the detection of the second, third and fourth acoustic peaks (Redhead et al. 2004b).

In addition to the temperature anisotropies, the anisotropies in polarization contain very relevant and independent information. In particular, in the standard model the maxima in the E spectrum are out of phase with those in the T spectrum due to the fact that polarized radiation is sensitive to the velocity of the fluid, and the velocity and density are out of phase in an acoustic wave. This shift is precisely what has been recently reported by Readhead et al. (2004b) based on CBI data (see figure 7 below). As we will see later, anisotropies in the polarization are very relevant to confirm the best fit model given by the temperature data and to constrain specific parameters as the optical depth to which they are very sensitive. 


\section{Cosmological parameters}

There are a number of cosmological parameters that account for very different fundamental physical properties of the universe and that influence the radiation angular power spectrum in many ways. These parameters characterize the background model of the universe (assumed to be the homogeneous and isotropic Friedmann-Robertson-Walker model), the primordial scalar and tensor fluctuations and the reionisation history. At present around 12 parameters are considered for the data analysis.

The parameters characterizing the background model of the universe are the following:

- Physical baryonic density, $w_{b}: w_{b}=\Omega_{b} h^{2}$.

- Physical matter density, $w_{m}: w_{m}=\Omega_{m} h^{2}$. $\Omega_{m}$ is given by the sum of the baryonic density $\Omega_{b}$, the CDM density $\Omega_{C D M}$ and the neutrino density $\Omega_{\nu}$.

- Physical neutrino density, $w_{\nu}: w_{\nu}=\Omega_{\nu} h^{2}$ (up to now only upper limits are found for this parameter).

- Dark energy equation of state parameter, $w: w \equiv p_{D E} / \rho_{D E}$.

- Dark energy density, $\Omega_{D E}$ : In case $w$ were constant and took the value -1 then the dark energy takes the form of a cosmological constant and its energy contribution is represented by $\Omega_{\Lambda}$.

- Hubble constant, $h: h \equiv H_{0} / 100 \mathrm{~km} \mathrm{~s}^{-1} \mathrm{Mpc}^{-1}$.

The reionisation history of the universe influences the CMB by a single parameter:

- Optical depth, $\tau: \tau=\sigma_{T} \int_{t_{r}}^{t_{0}} n_{e}(t) d t$, where $\sigma_{T}$ is the Thompson crosssection and $n_{e}(t)$ is the electron number density as a function of time.

The cosmological parameters that characterize the matter and gravitational waves primordial power spectra are:

- Amplitude of the primordial scalar power spectrum, $A_{s}$ : $P_{s}(k)=A_{s}\left(k / k_{0}\right)^{n_{s}}$, where $k_{0}=0.05 \mathrm{Mpc}^{-1}$.

- Scalar spectral index, $n_{s}$.

- Running index, $\alpha: \alpha=d n_{s} / d \ln k$. It accounts for the deviations from a pure power-law. Its value is normally determined at the scale $k_{0}=$ $0.05 \mathrm{Mpc}^{-1}$. 

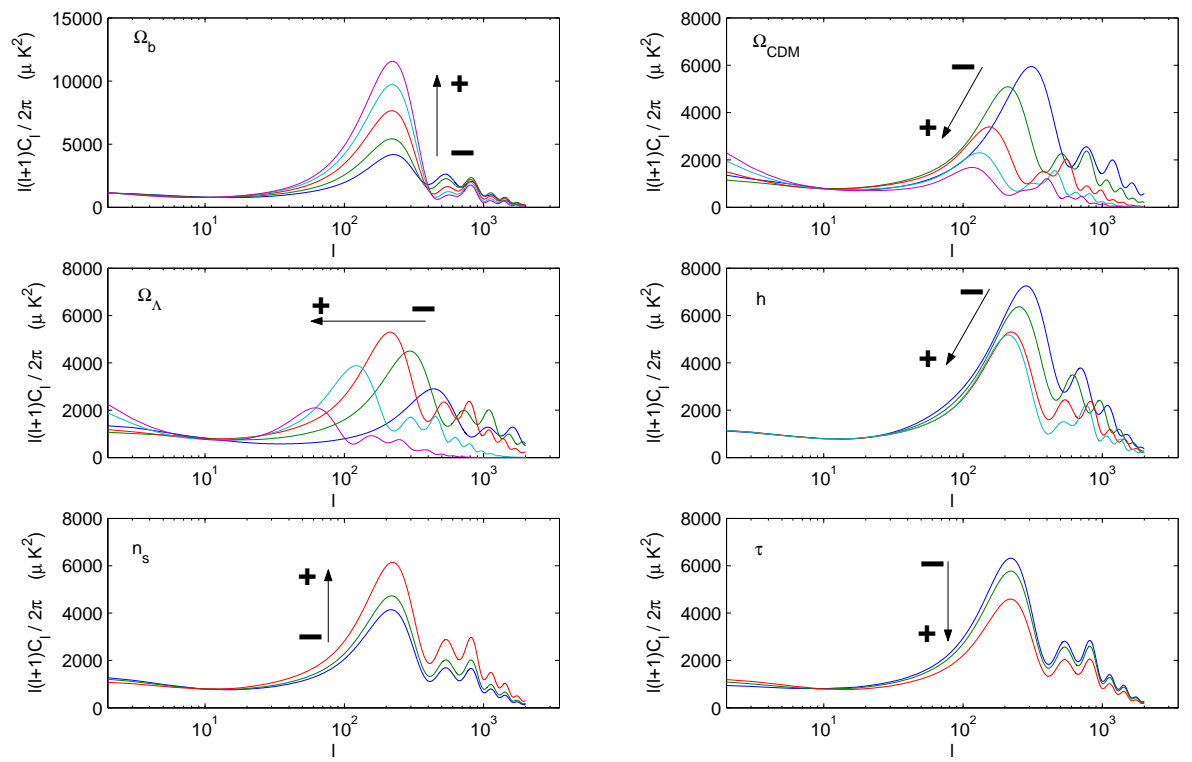

Figure 4. Dependence of $C_{\ell}$ on some relevant cosmological parameters (The $C_{\ell}$ has been produced with the CMBFAST code.

- Tensor-to-scalar ratio, $r: r=A_{t} / A_{s}$.

- Tensor spectral index, $n_{t}$ : from the consistency relation of inflation it is normally assumed that $n_{t}=-r / 8$.

Besides those parameters there are two possible types of primordial matter density fluctuations: adiabatic (the entropy per particle is preserved) and isocurvature (matter fluctuations compensate those of the radiation conserving total energy density). As commented in the Introduction the standard model of inflation predicts fluctuations of the adiabatic type.

Analyses that combine CMB with LSS require an additional parameter accounting for the bias $b$ of the galaxy density respect to the matter density.

In figure 4 we show the changes in $C_{\ell}$ produced by the variation of some of the most relevant cosmological parameters. The many different ways in which the $C_{\ell}$ can vary with the parameters produces degeneracies complicating their accurate determination. In particular, there is a well known geometric degeneracy involving the matter and dark energy densities. This is shown in figure 5 where almost identical angular power spectra are obtained for three different values of the curvature $\left(\Omega_{k}=1-\Omega_{m}-\Omega_{D E}\right)$. This example illustrates the need of including in the analysis additional cosmological data sets (like SN Ia, LSS, cluster density, CMB polarization, ...) to break the degeneracies. 


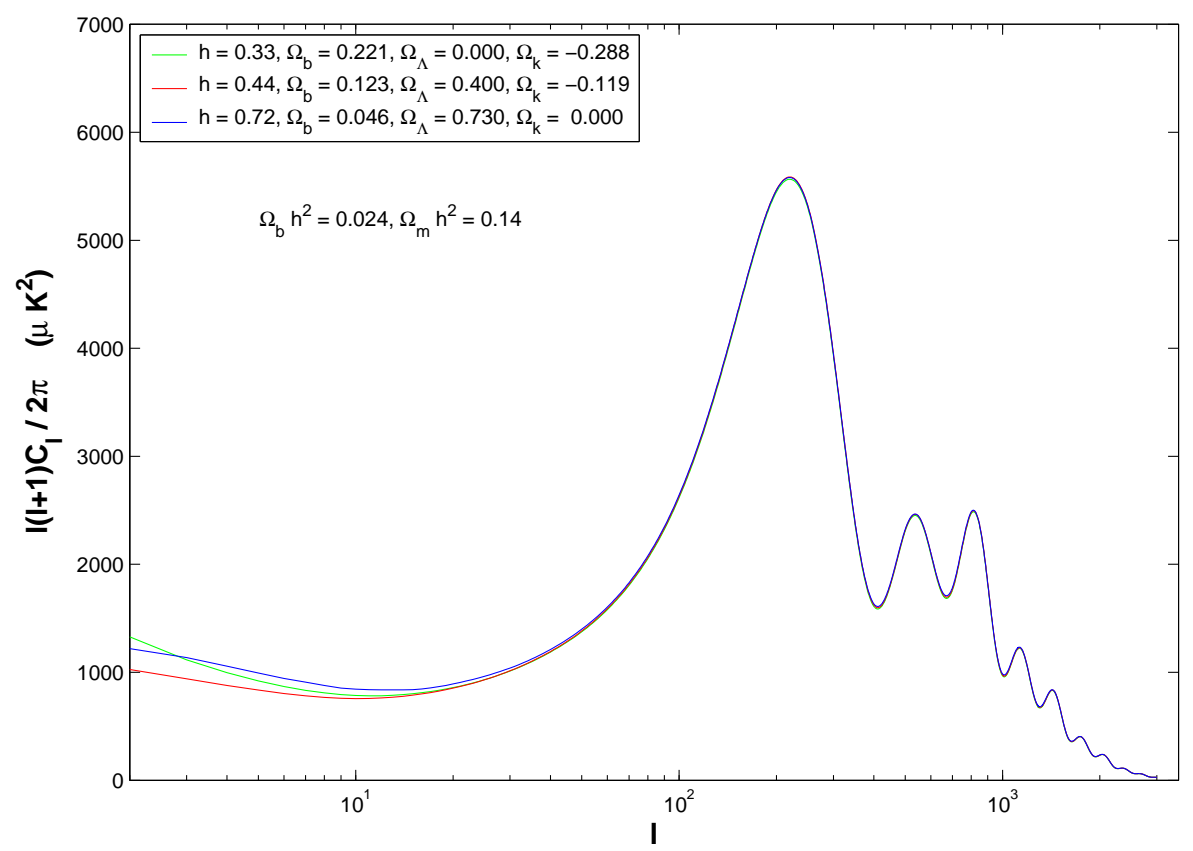

Figure 5. Geometric degeneracy for three values of the curvature $\Omega_{k}$. Apart from the parameters given in the figure the rest of the parameters have been fixed to the best fit model given in Bennett et al. (2003). The three spectra have been computed using the CMBFAST code.

\section{Cosmological constraints}

In recent years there has been an explosion of cosmological data which have made possible a strong advance in the determination of the cosmological parameters. Below we summarize the main results for $\mathrm{CMB}$ data alone and when combined with other cosmological data sets.

\section{WMAP and higher resolution CMB experiments}

The WMAP data alone is able to put strong constraints on some cosmological parameters when some priors are assumed in the analysis (Spergel et al. 2003). Table 1 summarizes the results when a flat universe is assumed, the prior $\tau<0.3$ is imposed on the optical depth and only 6 parameters are considered. It is interesting to notice that the Einstein-de Sitter universe (i.e. a spatially flat universe with null dark energy) is rejected at a very high confidence level. Besides, the value of the optical depth parameter $\tau$ is essentially determined by the TE angular cross-power spectrum.

The WMAP data also test the type of the primordial fluctuations. The clear detection of the first acoustic peaks as well as the detection of the TE cross- 


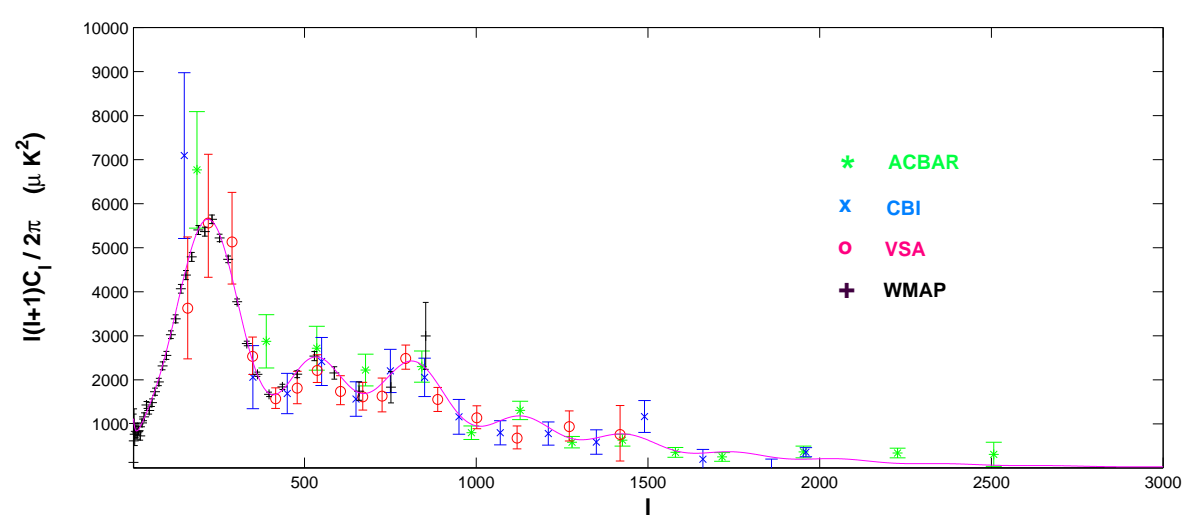

Figure 6. $C_{\ell}$ measured by WMAP, ACBAR, CBI and VSA, together with the best fit model given by Bennett et al. (2003). The values of the parameters are listed in table 2 .

correlation imply that the fluctuations were primarily adiabatic, in agreement with the standard inflationary model.

Table 1. Cosmological parameters using only WMAP data. In the fit the universe is assumed to be spatially flat and the value of the optical depth is constrained to $\tau<0.3$ (from Spergel et al. 2003)

\begin{tabular}{cc}
\hline Parameter & Values $(68 \% C L)$ \\
\hline$w_{b}$ & $0.024 \pm 0.001$ \\
$w_{m}$ & $0.14 \pm 0.02$ \\
$h$ & $0.72 \pm 0.05$ \\
$A_{s}$ & $0.9 \pm 0.1$ \\
$\tau$ & $0.166_{-0.071}^{+0.076}$ \\
$n_{s}$ & $0.99 \pm 0.04$ \\
\hline
\end{tabular}

The situation is improved if temperature data from high resolution CMB experiments is included in the analysis (Spergel et al. 2003, Dickinson et al. 2004). In figure 6 the $C_{\ell}$ obtained from the experiments WMAP (Hinshaw et al. 2003), ACBAR (Kuo et al. 2004), CBI (Readhead et al. 2004a) and VSA (Dickinson et al. 2004) is shown. Although the polarization measurements are not yet sensitive enough to significantly improve the constraints already derived from the temperature one (except for $\tau$ ), however the measured peaks in the E-mode $C_{\ell}$, which are out of phase with the temperature $C_{\ell}$ ones, suppose an independent evidence of the standard model and, more specifically of the adiabatic type of the primordial matter density fluctuations. The TE angular cross-power spectrum from DASI (Leitch et al. 2004), WMAP (Kogut et al. 
2003) and CBI (Readhead et al. 2004b) as well as the E-mode polarization angular power spectrum from DASI and CBI are shown in figure 7.
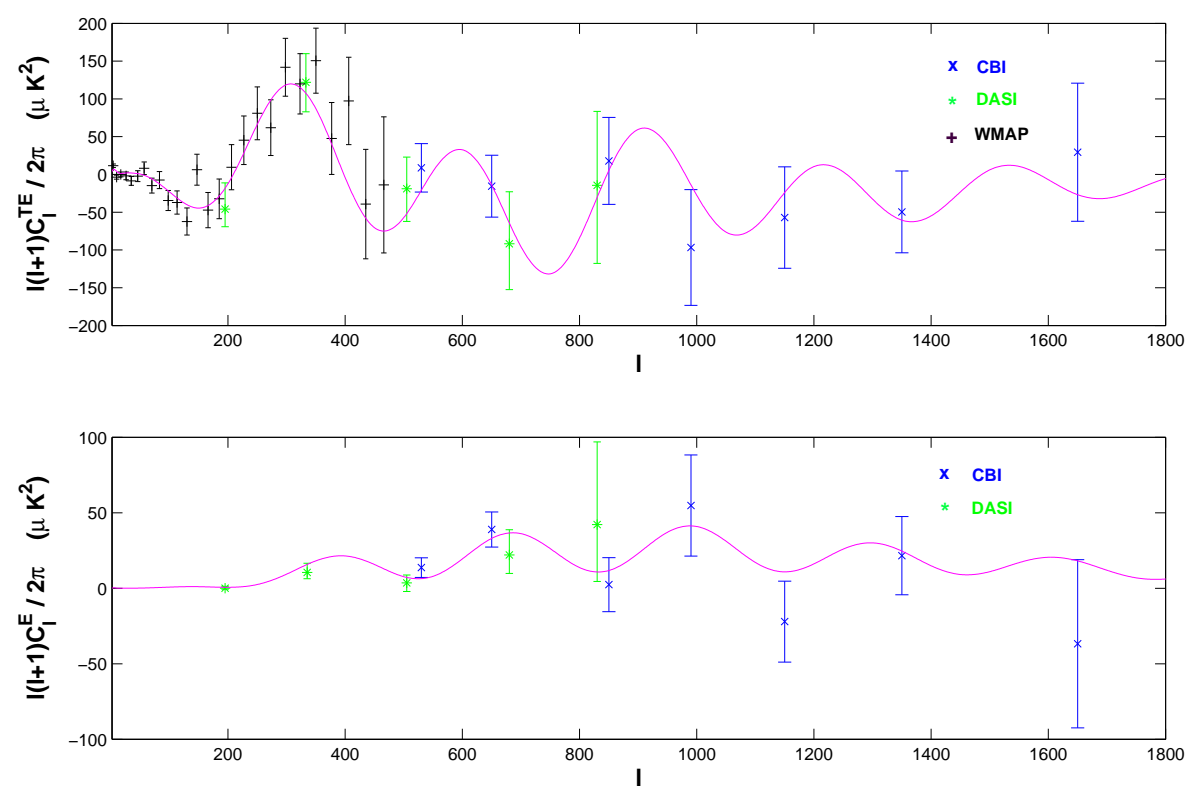

Figure 7. TE and E angular power spectra measured by DASI, WMAP and CBI (TE) and DASI and CBI (E). Also plotted is the best fit model given by Bennett et al. (2003). The values of the parameters are listed in 2 .

\section{Combination with other cosmological data sets}

The present richness of data is not exclusive of the CMB. Also large galaxy surveys covering a large fraction of the sky and measuring the three-dimensional power spectrum using $\approx 200000$ redshifts, like the 2 dFGRS (Percival et al. 2001) or SDSS (Tegmark et al. 2004a), have been recently achieved. If the initial fluctuations are Gaussian, all the information is included in the power spectrum $P(k)$ and thus this is the quantity normally used for the analysis. The cosmological parameters determine the matter power spectrum at present through the initial amplitude and the spectral index $A_{s}, n_{s}$ and the transfer function connecting linearly the present and initial $P(k)$. An additional bias parameter $b$, assumed to be scale-independent and linear, is needed to relate galaxy and matter density. Moreover, non-linear evolution of the matter density power spectrum should be also considered in the comparison with observations. The present matter density power spectrum as well as the luminosity distance-redshift diagram determined with SN Ia depend on the cosmological parameters in a very different way from the $C_{\ell}$, and so the combined data 
sets can potentially impose much more severe constraints. Results of combining CMB data with LSS galaxy surveys (Percival et al. 2001, Tegmark et al. 2004a), HST key project value of $H_{0}$ (Freedman et al. 2001), SN Ia magnitude-redshift data (Riess et al. 2001, Tonry et al. 2003), Ly $\alpha$ forest power spectrum (Croft et al. 2002, McDonald et al. 2004) are given in Bennett et al. (2003), Spergel et al. 2003, Tegmark et al. (2004b) and Seljak et al. (2004). As it is shown in these papers, the constraints on the cosmological parameters are greatly improved when combining all those cosmological data sets and, what is even more important, the best fit model is an acceptable fit for all of them. Table 2 shows the best fit cosmological parameters given in Bennett et al. (2003) for the combination WMAP+CBI+ACBAR+2dFGRS. Alternative combinations include WMAP, high redshift SN Ia and abundances of rich clusters of galaxies (see e.g. Rapetti, Steven and Weller 2005 and Jassal, Bagla and Padmanabhan 2005 for a detailed analysis of the constraints imposed by these data sets on the dark energy equation of state). In figure 8 confidence contours are given for the $\left(\Omega_{m}, \Omega_{D E}\right)$ plane combining WMAP, SN Ia (Knop et al. 2003) and galaxy cluster abundance (Allen et al. 2002). The complementarity of the three data sets is clearly noticed by the reduction of the contours of the combined data set compared to the individual ones.

Table 2. Cosmological parameters from WMAP, CBI, ACBAR and 2dFGRS combined data (from Bennett et al. 2003)

\begin{tabular}{cc}
\hline Parameter & Values $(68 \% \mathrm{CL})$ \\
\hline$w_{b}$ & $0.0224 \pm 0.0009$ \\
$w_{m}$ & $0.135_{-0.009}^{+0.009}$ \\
$w_{\nu}$ & $<0.0076(95 \% \mathrm{CL})$ \\
$w$ & $<-0.78(95 \% \mathrm{CL})$ \\
$\Omega_{D E}$ & $0.73 \pm 0.04$ \\
$h$ & $0.71_{-0.03}^{+0.04}$ \\
$\tau$ & $0.17 \pm 0.04$ \\
$A_{s}$ & $0.833_{-0.083}^{+0.086}$ \\
$n_{s}$ & $0.93 \pm 0.03$ \\
$\alpha$ & $-0.031_{-0.018}^{+0.016}$ \\
$r$ & $<0.90(95 \% \mathrm{CL})$ \\
\hline
\end{tabular}

\section{Integrated Sachs-Wolfe effect}

There are strong evidences that the universe today is dominated by the dark energy density $\Omega_{D E}$. The evidences came first from measurements of the luminosity curve and redshift of distance SN Ia, and later from surveys of the CMB anisotropy and LSS distribution of galaxies. More recently there have 


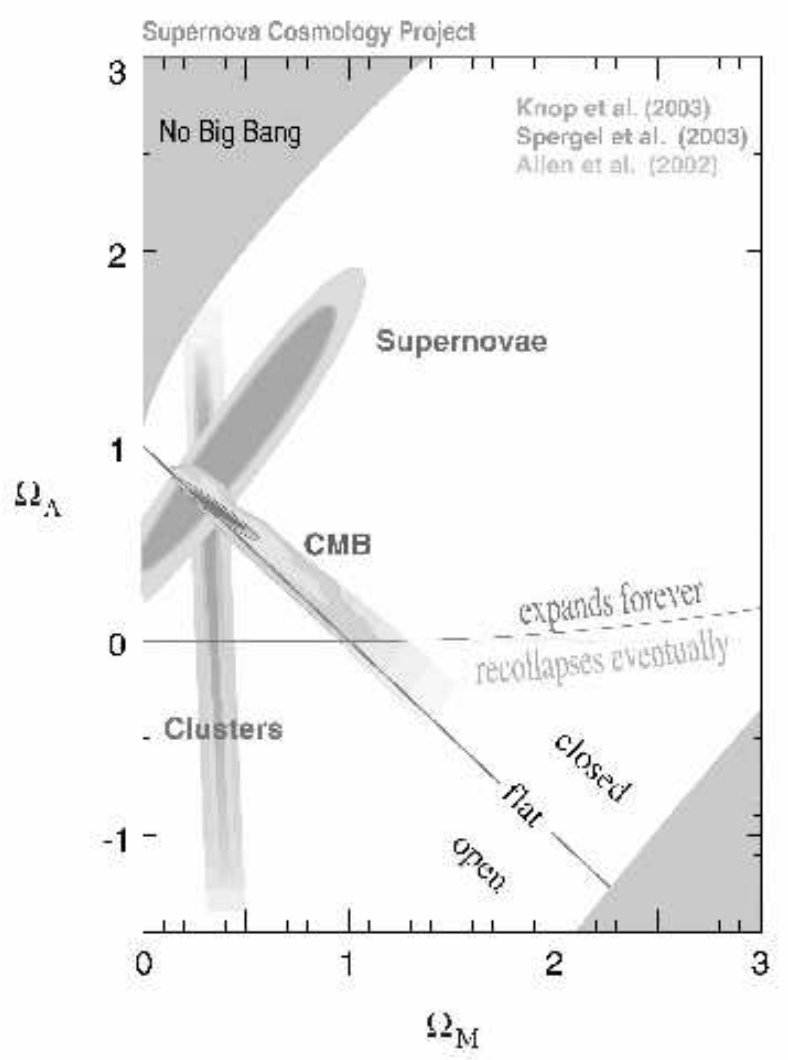

Figure 8. Confidence contours for the plane $\left(\Omega_{m}, \Omega_{\Lambda}\right)$ using SN Ia, CMB and cluster density data (taken from the Supernova Cosmology Project).

been independent tests that confirm this result. They come from the crosscorrelation of the CMB map with LSS surveys which span a wide range in redshift. From the late ISW effect a non null signal is expected due to the fact that the same gravitational potential created by the LSS at late times is also leaving an imprint on the CMB anisotropies. The amplitude and sign of the effect are determined by $\Omega_{D E}, \Omega_{k}$ and $h$. If the universe is close to spatially flat, as found in a consistent way by very different cosmological data sets, then a positive LSS-CMB cross-correlation would be an unambiguous indication of the presence of dark energy.

This is what has been recently found by a number of authors using different surveys and methods (Boughn and Crittenden 2004; Fosalba, Gaztañaga and Castander 2004; Nolta et al. 2004; Afshordi, Loh and Strauss 2004; Vielva, 


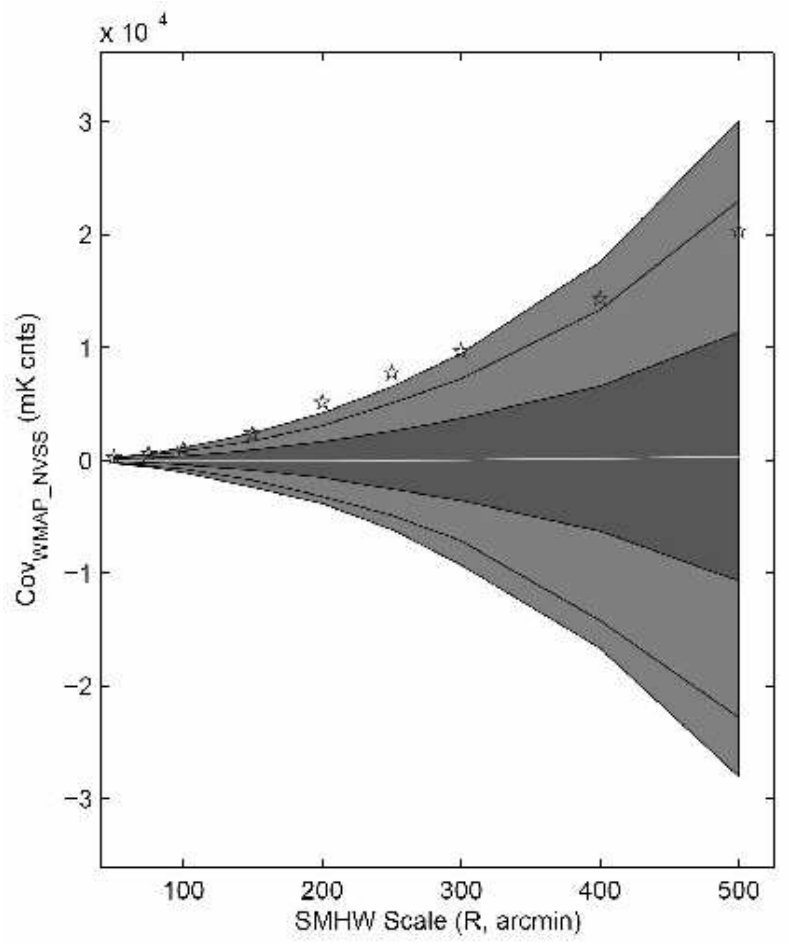

Figure 9. Detection of the ISW effect, above $3 \sigma$ level, applying spherical wavelets to the NVSS and WMAP maps and computing the covariance of the wavelet coefficients as a function of the wavelet scale. The contours are 1,2 and $3 \sigma$ (from Vielva, Martínez-González and Tucci 2004).

Martínez-González and Tucci 2004). The maximum amplitude for the signal has been obtained in the latter paper by cross-correlating the radio galaxy survey NRAO VLA Sky Survey (NVSS, Condon et al. 1998) with the WMAP data. It represents a detection $>3 \sigma$ (see figure 9).

\section{Open problems}

Looking at table 2 and considering the situation only ten years ago, the progress in the determination of the cosmological parameters has been spectacular. However, what is even more impressive is the concordance among all cosmological data sets available at present. This agreement in the results has made possible to build a well specified model of the universe, the so called concordance model. Nevertheless there are still pending problems related to the data fitting as well as the interpretation of the values of some of the param- 
eters, whose solutions will need to wait for future more sensitive experiments (at least for some of them, see below).

In spite of the very precise overall fit of the theoretical $C_{\ell}$ to the WMAP data (Spergel et el. 2003), there are some significant discrepancies. There are also significant deviations from the assumed isotropic Gaussian random field hypothesis, property which is also assumed in the parameter estimation. Moreover, some parameters take uncomfortably high values and the nature of others is completely unknown. More specifically, some of the most relevant open problems are:

- Anomalies at the lowest multipoles $(\ell=2,3)$ : deficit of power and the coincidence of orientations of the quadrupole and octopole in the WMAP data (Bennett et al. 2003, de Oliveira-Costa et al. 2004, Efstathiou et al. 2004). A better removal of the Galactic contamination narrowing the mask used for the analysis (as expected from the future Planck mission, see below) can help to clarify this situation. An attempt to solve this problem invokes a global non-trivial topology for the universe (Luminet et al. 2003).

- Anomalies at intermediate multipoles $(\ell=22,40,210)$ : glitches have been found around those multipoles in the WMAP data (Bennett et al 2003). It is not clear if they are just statistical fluctuations, foreground contamination or represent real features. Some of them might be related to the asymmetries discussed below (Hansen et al. 2004).

- Asymmetries and non-Gaussianity: a number of authors have found significant deviations from isotropy and Gaussianity in the WMAP data. In particular asymmetries along the sky and/or non-Gaussianity have been found in the genus (Park 2004), low-order moments (Eriksen et al. 2004, Hansen et al. 2004), wavelets (Vielva et al. 2004, Mukherjee and Wang 2004), phases (Chiang et al. 2003), extrema (Larson and Wandelt 2004), directional wavelets (McEwen et al. 2005) and local curvature (Cabella et al. 2005). In addition a very cold spot has been detected and localized (Cruz et al. 2005). Although some of these results could be due to foregrounds others seem to be very difficult to explain in that way. Anisotropic models for the universe of the type Bianchi $\mathrm{VII}_{h}$ with very small vorticity and shear seem to provide a good fit to all those features (Jaffe et al. 2005); however they require a total $\Omega=0.5$, which deviates significantly from the value of the concordance model.

- Power excess at high multipoles ( $\ell>2000$ ): several high resolution experiments have found excess of power at multipoles $2000<\ell<3000$ (CBI, DASI, ACBAR). This excess can be associated, at least partially, 
to SZ emission from galaxy clusters (Bond et al. 2005) or to extragalactic sources (Toffolatti et al 2005). Diego et al. (2004) suggested a sensitive way to separate between those possibilities using the skewness and kurtosis of the Mexican Hat wavelet coefficients.

- High value of the optical depth: from the WMAP data a high value of the Thompson optical depth is derived ( $\tau \simeq 0.17$, see tables 1 and 2 ), implying a high redshift for the reionization of the inter-galactic medium of the universe $z \sim 20$ and, thus, that non-linear structures were already formed at these high redshifts. This result heavily relies on the high value of the TE angular cross-power spectrum at low multipoles measured by WMAP, and so a confirmation with the second and other years of data - particularly with the E-mode angular power spectrum — would be crucial. Again, sensitive polarization data covering a large fraction of the sky (as expected from Planck) would represent an important step forward to solve this problem.

- Non-zero running index $\alpha$ : WMAP data combined with other cosmological data sets slightly favours a running index different from zero (see table 2 . This result is heavily supported by the data at small scales provided by the Ly $\alpha$ forest power spectrum (Croft et al. 2002). However, new analyses of the matter power spectrum from the Ly $\alpha$ forest find values of $\alpha$ perfectly consistent with zero (Seljak et al. 2005).

- Isocurvature fluctuations: Although the WMAP temperature and polarization data imply that the primordial density fluctuations were primarily adiabatic (also confirmed by the recent polarization measurements from $\mathrm{CBI}$ ), however at the $2 \sigma \mathrm{CL}$ up to $\approx 50 \%$ of the $C_{\ell}$ power can still be due to isocurvature fluctuations (Crotty, Lesbourgues and Pastor 2003). In addition, considering all possible combinations of adiabatic and isocurvature modes for each matter component will significantly lower the constraints in the parameters (Bucher, Moodley and Turok 2001). Future sensitive experiments like Planck will be able to further constraint the type of the fluctuations and their relative amounts.

- Topological defects: the acoustic oscillations measured in the $C_{\ell}$ by WMAP and other CMB experiments strongly reduce the possible role of topological defects in the universe. Recently, Bevis, Hindmarsh and Kunz (2004) and Fraisse (2005) have limited the contribution of topological defects (textures and cosmic strings) to the $C_{\ell}$ to be $\lesssim 20 \%$. However, a fine determination of their possible sub-dominant contribution will probably have to wait for future more precise data.

- Nature of dark matter and dark energy: probably the most challenging problem in cosmology is to understand the nature of the dark en- 
ergy and the dark matter which completely dominates the dynamics of the universe. For the former, a cosmological constant (with equation of state $w=-1$ ) seems to be in acceptable agreement with many different data sets. This situation is particularly embarrassing since a natural explanation in terms of the vacuum energy, given by quantum zero-point fluctuations of fundamental fields, implies a value around 100 orders of magnitude above the observed one. Regarding the dark matter, although several candidates have been proposed within particle physics theories, neither its nature nor its evolutionary history are known.

- B-mode polarization: detection of this mode would unambiguously indicate the existence of a primordial background of gravitational waves and thus would allow to constrain theories of the early universe. Due to its expected weak signal respect to the temperature, the E-mode anisotropies (see figure 3) and to the emission from the Galactic and extragalactic foregrounds (Tucci et al. 2005), its detection and precise determination will necessarily require the development of new very sensitive experiments.

On top of these problems there are others, more fundamental ones, related to the origin of inflation and to its dynamics: which is the particle physics framework for inflation?, how the universe started to inflate in the early universe?, which is the specific model of inflation within, e.g., the simple classification by Kinney (2002)? The ESA Planck mission, the third space mission after COBE and WMAP, scheduled for launch in 2007, will measure the temperature of the $\mathrm{CMB}$ over the whole sky with high sensitivity (few $\mu \mathrm{K}$ ), resolution (down to 5 arcmin) and wide frequency coverage $(30-850 \mathrm{GHz})$, allowing a good separation between $\mathrm{CMB}$ and foregrounds and thus providing an angular power spectrum with unprecedented precision limited only by cosmic variance. It will also provide a map of polarization with sensitivity beyond the one reached by previous experiments. These new measurements will certainly have a profound impact on our understanding of the origin and evolution of our universe (for more details on the Planck mission see the web page address given in the references). An open issue which may remain even after Planck is the detection of the B-mode polarisation. As stated above, the determination of this mode would have a tremendous impact on the theories of the early universe. There are now many plans to build experiments capable to reach the demanding sensitivities needed to search for the B-mode including space missions from ESA and NASA.

Finally, from the previous discussion we can state that the role played by the $\mathrm{CMB}$ on our understanding of the universe has been very relevant in the past and is expected to continue being so in the future. 


\section{Acknowledgments}

We thank R.B. Barreiro for useful comments on the manuscript. We acknowledge financial support from the Spanish MEC project ESP2004-07067C03-01. We also acknowledge the use of LAMBDA, support for which is provided by by the NASA Office of Space Science. We acknowledge the use of the software package CMBFAST (http://www.cmbfast.org) developed by Seljak and Zaldarriaga. The work has also used the software package HEALPix (http://www.eso.org/science/healpix) developed by K.M. Gòrski, E.F. Hivon, B.D. Wandelt, J. Banday, F.K. Hansen and M. Barthelmann.

\section{References}

Afshordi N., Loh Y.-S. \& Strauss M. A., 2004, Phys. Rev. D, 69, 3524

Allen A.W., Schmidt R.W. \& Fabian A.C., 2002, MNRAS, 334, L11

Barreiro R.B., 2000, New Astronomy Reviews, 44, 179

Bartolo N., Komatsu E., Matarrese S. \& Riotto A., 2004, Phys. Rep., 402, 103

Bennett C. L. et al., 2003, ApJS, 148, 1

Bersanelli M., Maino, D. \& Mennella A., 2002, Nuovo Cimento, 25, 1

Bevis N., Hindmarsh M. \& Kunz M., 2004, Phys. Rev. D, 70, 043508

Bond et al., 2005, ApJ, 626, 12

Bouchet F.R. \& Gispert R., 1999, New Astronomy Reviews, 4, 443

Boughn S. P. \& Crittenden R. G., 2004, Nature, 427, 45

Bucher M., Moodley K. \& Turok N., 2001, Phys. Rev. Lett., 87, 191301

Cabella, P. \& Kamionkowski, M., 2004, astro-ph/0403392

Cabella P., Liguori M., Hansen F.K., Marinucci D., Matarrese S., Moscardini L. \& Vittorio N., 2005, MNRAS, 358, 648

Cruz M., Martínez-González E., Vielva P. \& Cayón L., 2005, MNRAS, 356, 29

Challinor A., 2004, astro-ph/0403344

Challinor A., 2005, astro-ph/0502093

Chiang L.-Y., Naselsky P.D., Verkhodanov O.V. \& Way M.J., 2003, ApJL, 590, 65

Condon J.J. et al., 1998, AJ, 115, 1693

Croft R.A.C. et al., 2002, ApJ, 581, 20

Crotty P., Lesbourgues J. \& Pastor S., 2003, Phys. Rev. D, 67, 123005

De Bernardis P. et al., 2000, Nature, 404, 955

de Oliveira-Costa A., Tegmark M., Zaldarriaga M. \& Hamilton A., 2004, Phys. Rev. D, 69, 063516

Delabrouille J., Cardoso J.-F. \& Patanchon G., 2003, MNRAS, 346, 1089

Dickinson C. et al., 2004, MNRAS, 353, 732

Diego J.M., Martínez-González E., Vielva P. \& Silk J., 2004, astro-ph/0403561

Efstathiou G., 2004, MNRAS, 348, 885

Eriksen H.K., Hansen F.K., Banday A.J., Gorski K.M. \& Lilje P.B., 2004, ApJ, 605, 14

Fosalba P., Gaztañaga E. \& Castander F., 2004, ApJL, 597, 89

Fraisse A.A., 2005, Phys. Rev. Lett., submitted, astro-ph/0505402

Freedman W.L. et al., 2001, ApJ, 553, 47

Hanany S. et al., 2000, ApJ, 545, L5 
Hansen F.K., Banday A.J. \& Gòrski K.M., 2004, MNRAS, 354, 641

Herranz D., Sanz J.L., Hobson M.P., Barreiro R.B., Diego J.M., Martínez-González E. \& Lasenby A.N., 2002, MNRAS, 336, 1057

Hinshaw G. et al., 2003, ApJ, 148, 135

Hu W. and Dodelson S., 2002, ARAA, 40, 171

Hobson M.P., Jones A.W., Lasenby A.N. \& Bouchet F., 1998, MNRAS, 300

Jaffe T.R., Banday A.J., Eriksen H.K., Gorski K.M. \& Hansen F.K.,2005, ApJL, submitted, astro-ph/0503213

Jassal H.K., Bagla J.S. \& Padmanabhan T., 2005, astro-ph/0506748

Kamionkowski M., Kosowsky A. \& Sttebins A., 1997, Phys. Rev. D, 55, 7368

Kinney W.H., 2002, Phys. Rev. D, 66, 083508

Knop R.A. et al., 2003, ApJ, 598, 102

Kogut A. et al., 2003, ApJS, 148, 161

Kovac J.M. et al. 2002, Nature, 420, 772

Komatsu E. et al., 2004, ApJS, 148, 119

Kuo C.L. et al., 2004, ApJ, 600, 32

Larson D.L. \& Wandelt B.D., 2004, ApJ, 613, 85

Leitch et al., 2004, ApJ, submitted, astro-ph/0409357

Luminet J.-P., Weeks J.R., Riazuelo A., Lehoucq R. \& Uzan J.-P., 2003, Nature, 425, 593

Maino D. et al., 2002, MNRAS, 334,53

Martínez-González E., Sanz J.L. \& Silk, 1990, ApJ, 335, 5

Martínez-González E., Diego J.M., Vielva P. \& Silk J., 2003, MNRAS, 345, 1101

Mather J.C. et al. 1994, ApJ, 420, 439

Mather J.C. et al. 1999, ApJ, 512, 511

McDonald P. et al., 2004, ApJ, submitted (astro-ph/0407377)

McEwen J. D., Hobson M. P., Lasenby A. N. \& Mortlock D. J., 2005, MNRAS, 359, 1583

Mennella A. et al., 2004, astro-ph/0402528

Mukherjee P. \& Wang Y., 2004, ApJ, 613, 51

Nolta M. R. et al., 2004, ApJ, 608, 10

Park C.-G., 2004, MNRAS, 349, 313

Percival W.J. et al., 2001, MNRAS, 327, 1297

Planck mission, http://www.rssd.esa.int/Planck

Rapetti D., Steven S.W. \& Weller J., 2005, MNRAS, 360, 555

Readhead A.C.S. et al., 2004a, ApJ, 609, 498

Readhead A.C.S. et al., 2004b, Science, 306, 836

Riess et al., 2001, ApJ, 560, 49

Sachs R. K. \& Wolfe A. M., 1967, ApJ, 147, 73

Sanz J.L., 1997, In The Cosmic Microwave Background, eds. C.H. Lineweaver et al., Kluwer Academic Publishers, p. 33

Scott D.H., Srednicki M. \& White M., 1994, ApJ, 421, 5

Seljak U. \& Zaldarriaga M., 1996, ApJ, 469, 437

Seljak U. et al., 2005, Phys. Rev. D, 71, 103515

Silk J., 1968, ApJ, 151, 459

Smoot G. F. et al., 1992, ApJL, 396, L1

Spergel D. N. et al., 2003, ApJS, 148, 175

Sunyaev R.A. \& Zeldovich Y.B., 1972, Comm. Astrophys. Space Phys., 4, 173 
Tegmark M., Eisenstein D.J., Hu W. \& de Oliveira-Costa A., 2000, ApJ, 530, 133

Tegmark M. et al., 2004a, ApJ, 606, 702

Tegmark M. et al., 2004b, Phys. Rev. D, 69, 103501

Tonry J.L. et al., 2003, ApJ, 594, 1

Tucci M., Martínez-González E., Vielva P. \& Delabrouille J., 2005, MNRAS, 360, 935

Toffolatti L., Negrello M., González-Nuevo J., de Zotti G., Silva L., Granato G.L. \& Argüeso F., 2004, A\&A, in press, astro-ph/0410605

Vielva P., Barreiro R. B., Hobson M. P., Martínez-González E., Lasenby A. N., Sanz J. L. \& Toffolatti L., 2001, MNRAS, 328, 1

Vielva P., Martínez-González E., Barreiro R.B., Sanz J.L. \& Cayón L., 2004, ApJ, 609, 22

Vielva P., Martínez-González E. \& Tucci M., 2004, MNRAS, submitted, astro-ph/0408252

Watson R.A., Rebolo R., Rubiño-Martín J.A., Hildebrant S., Gutiérrez C.M., Fernádez-Cerezo S., Hoyland R.J. \& Battistelli E.S., 2005, ApJ, submitted, astro-ph/0503714

Zaldarriaga M. \& Seljak U., 1997, Phys. Rev. D, 55, 1830 